\section{Society of Hair Testing}

\author{
A. M. Gressner ${ }^{1}$ und O. A. Gressner ${ }^{2}$ \\ ${ }^{1}$ Labor Dr. Wisplinghoff Berlin, Berlin, Deutschland \\ ${ }^{2}$ Labor Dr. Wisplinghoff Köln, Köln, Deutschland
}

Synonym(e) Gesellschaft für Haaranalytik; SoHT

Definition Internationale Gesellschaft zur Förderung der Technologie und Analytik von Haarproben sowie deren wissenschaftliche und klinische Bewertungen.

Beschreibung Die 1995 gegründete internationale Gesellschaft widmet sich der Förderung der Forschung und Entwicklung zur Analytik von Haaren für diagnostische, foren- sische und arbeitsmedizinische Zwecke. Damit verbunden sind entsprechende Analysen zum Nachweis von Dopingpharmaka und zur Drogenkonsumption. Zu diesem Zweck werden von der SoHT regelmäßig aktuelle ConsensusStatements publiziert und internationale Kongresse zur Präanalytik, Technologie, Materialgewinnung und Interpretation der Ergebnisse organisiert.

\section{Literatur}

Cooper GAA, Kronstrand R, Kintz P (2012) Society of hair testing guidelines for drug testing in hair. Forensic Sci Int 218(1-3):20-24

Europ Workplace Drug Testing Society, edit. (2010) Drug and alcohol testing in hair, collection and analysis. Version 1.0

http://www.soht.org/ 